\title{
Arterio-venous Fistula for Dialysis- Procedure, Complications and Outcome
}

\author{
Bhawna Prabhakar ${ }^{1}$, Sanjay Sadhu², Tanuj Bhatia ${ }^{3}$ \\ ${ }^{1}$ Assistant Professor, ${ }^{2}$ Associate Professor, Department of Plastic and Reconstructive Surgery, ${ }^{3}$ Associate \\ Professor, Department of Cardiology, SGRRIHMS and SMI Hospital, Dehradun
}

\begin{abstract}
Background- In India, a 2018 estimate put the number of patients on chronic dialysis at about 175,000, giving a prevalence of 129 per million population. About two thirds of all patients with kidney failure die without receiving dialysis every year. For the other third, the majority (64.2\%) undergo renal replacement therapy via hemodialysis. AV fistulas are the preferred method for vascular access for hemodialysis. This study describes the outcome of $634 \mathrm{AV}$ fistulas created in our institute between January 2015 and December 2020.
\end{abstract}

Methods- 634 patients who underwent AV fistula creation in 5 years were retrospectively studied. Evaluation criteria were patency of AV Fistula at 1 week, 1 month, 6 months, 1 year and 2 years after creation; suturing technique- continuous versus interrupted, 6-0 versus 8-0 suture; end-to-side versus side-to-side anastomosis, mean operating time; duration of hospital stay; and complications- minor bleeding episodes, major bleeding requiring intervention, serous discharge, hyperaemia, upper limb swelling, aneurysm of anastomosis and recipient vein, delayed rupture.

Conclusions- Patency rate of AV Fistula at 1 week was $91.2 \%$, at 1 month was $84.6 \%$, at 6 months was $83.8 \%$ and at 1 year was $82.9 \%$, after creation. After 2 years $74.8 \%$ AVF's were still useful for dialysis. Factors affecting patency were location of anastomosis, low-dose Aspirin and regular dialysis

Key Words- AV Fistula, Vascular access for dialysis, Radio-cephalic AV Fistula, Brachio-cephalic AV Fistula

\section{Introduction}

In India, prevalence of End Stage Renal Disease shows the Iceberg phenomenon. Data on incidence and prevalence of kidney failure remain estimates because there are no kidney failure registries. The Million Death Study estimated the number of kidney failure deaths to be 136,000 in 2015 . A 2018 estimate put the number of patients on chronic dialysis in India

\section{Corresponding author:}

Dr Bhawna Prabhakar

Department of Plastic and Reconstructive Surgery, SGRRIHMS and SMI Hospital, Dehradun

Email: prabhakarbhawna@gmail.com at about 175,000 , giving a prevalence of 129 per million population $\left({ }^{1)}\right.$. A systematic review estimated that about two thirds of all patients with kidney failure died without receiving dialysis in 2010 . For the other third that actually have access to healthcare facilities, there is initially a hesitation to accept regular renal replacement therapy. There is a misconception in the Indian population that Renal replacement therapy rather than End Stage Renal Disease(ESRD) itself is the cause of morbidity and mortality. Add to that, a reliance on archaic systems of medicine that are supposedly foolproof and without complications, even in the so-called developed strata of the society. Our healthcare delivery systems have a long way to 
go before modern, evidence-based medicine gets its due acceptance.

The majority (64.2\%) of ESRD patients undergo renal replacement therapy via hemodialysis. Less frequently used renal replacement therapy treatments are renal transplant $(29.3 \%)$ and peritoneal dialysis $\left.(6.4 \%){ }^{1}\right]$. The methods of delivering hemodialysis include tunneled and non-tunneled catheters, arteriovenous grafts (AVGs), and arterio-venous fistulas.

Among the hemodialysis delivery options, central venous catheters have the highest rates of thrombosis and infection. Additionally, blood flow rates from hemodialysis catheters are typically the lowest of all hemodialysis access types. Finally, hemodialysis catheters are associated with greater mortality than AVG's or AVF's [3]. AV Fistulas are preferred over AV Grafts as AVF's have lower rates of thrombosis, have longer lifespans, and maintain their patency with fewer secondary interventions and a lower cost compared with grafts. Infection rates, rates of steal syndrome, and rates of symptomatic central venous stenosis are lower in patients with AVF's than AVG's. Most importantly, there is an approximate $20 \%$ decreased mortality rate in ESRD patients dialyzed through fistulas compared with those dialyzed through grafts $\left[^{2}\right]$.

There are three main types of AVF's. Radiocephalic fistula is a forearm fistula created by anastomosing the side of radial artery to the end of cephalic vein. Brachio-cephalic fistula is created in at the elbow by connecting the side of brachial artery to the end of cephalic vein. Finally, the brachial arteryto-transposed basilic vein (BTB) fistula is created by anastomosing the side of brachial artery to the end of basilic vein that has been transposed laterally and elevated superficially to make it amenable to dialysis cannulation $\left[{ }^{8}\right]$.

\section{Materials and Methods}

This study, conducted in our tertiary care hospital, includes all cases of arterio-venous fistula creation from January 2015 to December 2020. Clinical mapping of upper limb vessels and Colour Doppler ultrasonography were done in the non-dominant upper limb to choose a site for arterio-venous fistula creation. A peripheral vein is considered suitable to become a recipient vein for an $\mathrm{AV}$ fistula if it has a diameter of at least $1.5 \mathrm{~mm}$ with minimal branching pattern, a more or less straight course and is superficial enough to be either visible or palpable or both on applying pressure proximal to the intended site for AV fistula creation. A vein that does not satisfy at least the last of these criteria will not become a satisfactory recipient vein, even if it is visible in a Colour Doppler scan. All patients of ESRD who required long term hemodialysis and had vessels suitable for the creation of AV fistula were included in the study. Patients of ESRD in whom suitable recipient vein and artery in proximity were not available in either upper limb, after detailed clinical examination and Doppler mapping or patients who are not hemo-dynamically stable, were not included.

Procedure- Patients were asked to have a light meal before the surgery and also have their medication including anti-hypertensives. The treating nephrologist was requested to schedule a hemodialysis a day prior to surgery, especially in patients with orthopnoea and those on twice or thrice weekly dialysis. Patients were operated on in supine position with arm outstretched. Patients with a history of orthopnoea had to be operated on in sitting position. CRF patients are usually on some anti-platelet medication. This was stepped down to a single anti-platelet 3-4 days prior to the surgery for AVF creation.

A cocktail of Inj. Lidocaine(2\%) $4 \mathrm{ml}$ and Inj. Bupivacaine $(0.5 \%) 4 \mathrm{ml}$ was injected in the local site after marking superficial veins and the donor artery. 
This provides pain relief for 5-6 hours. End to side anastomosis was the most common technique used. Few cases of side to side anastomosis were also done but this technique had to be abandoned because of the occurrence of distal oedema in nearly all such cases. Prolene 6-0 was used for Brachio-cephalic and Brachio-basilic AVF creation as a continuous suture. Ethilon 8-0 was used in the form of interrupted sutures for Radio-cephalic AVF.

Drains were not routinely placed. Only in few cases where a serosanguinous collection was anticipated, glove drains were used. A total of 361 patients (57.2\%) were given low dose aspirin(75-150 mg OD) from the day of surgery, to be continued under guidance of the treating nephrologist.

In case a patient needs routine HD 48-72 hours after surgery, heparin free HD should be done, otherwise minor or major bleeding episodes can occur from the surgical site. In such cases, the effect of heparin can be offset using Protamine sulphate in a dose titrated according to the amount of heparin used. Mild blood discharge was managed conservatively with frequent dressing changes. Excessive bleeding episodes required intervention.

AVF functions better with Systolic blood pressure towards the higher side, accordingly the dose of antihypertensives needs to be adjusted in conjunction with the nephrologist. Paediatric patients have higher rates of AVF closure but sufficient number of cases are not available to do a statistical analysis. Patients were asked to elevate the operated limb for at least 3 weeks after the surgery. Isotonic and isometric exercises of forearm muscles and hand were encouraged as frequently as possible. The patients were also educated about how to feel the AVF thrill. In case, the thrill was feeble or absent, they were asked to report to the hospital as soon as possible. After a Doppler scan, they were then referred to the cardiologist for possible intervention.

Evaluation criteria-

Suturing technique- continuous versus interrupted, 6-0 Prolene versus 8-0 Ethilon

End-to-side versus side-to-side anastomosis

\section{Mean operating time}

Patency of AV Fistula at 1 week, 1 month, 6 months, 1 year and 2 years after creation

\section{Duration of hospital stay}

Complications- Minor bleeding episodes, major bleeding requiring intervention, Serous discharge, Hyperemia, Upper limb swelling, Aneurysm of anastomosis and recipient vein, Delayed rupture

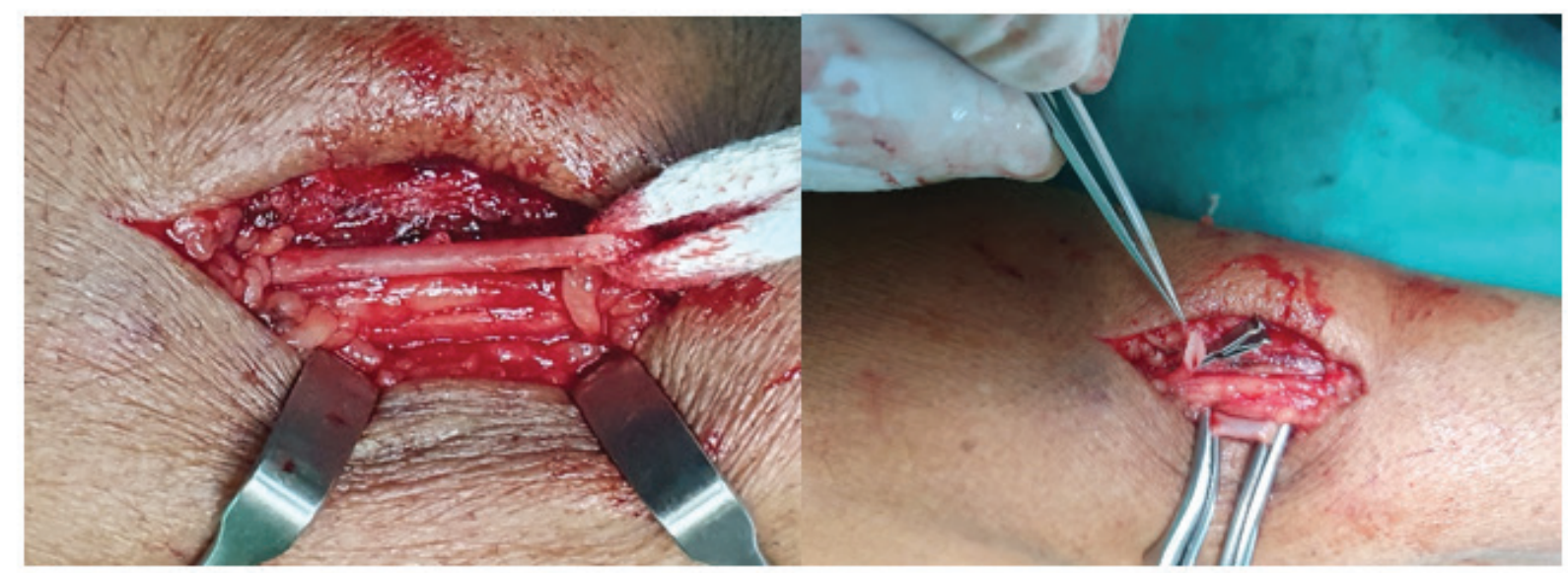




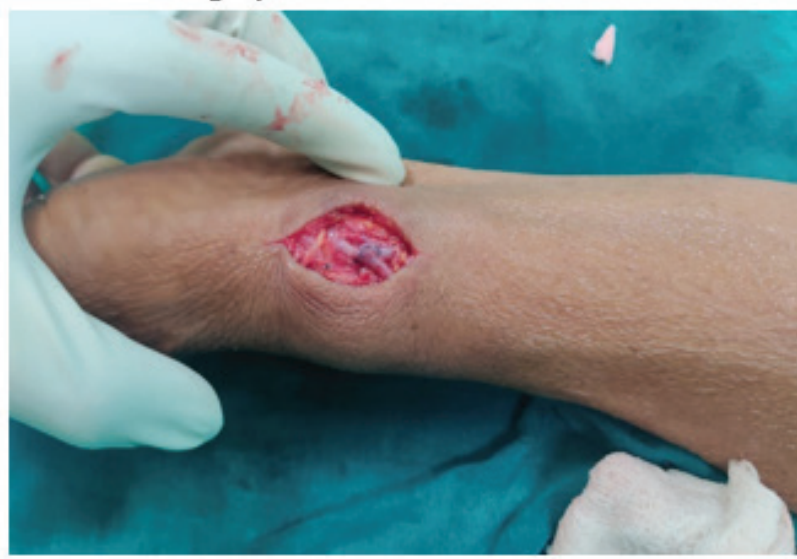

Clinical Photograph 3

Clinical photographs showing (1) Dissected Cephalic vein in distal forearm, (2) Cut and clamped Cephalic vein and Clamped Radial artery in distal forearm, (3) Left distal forearm Radio-cephalic AVF after anastomosis, (4) Left Brachio-cephalic AVF after anastomosis

Observations: Most patients were males (69.7\%). The mean age was 47.9 years. Comorbidities seen included diabetes mellitus (40.4\%), hypertension (89.6\%), hypertension and diabetes mellitus (21.2\%). Out of 634 patients, 119 patients $(18.8 \%)$ had previous arterio-venous fistula failure, 534 patients $(84.3 \%)$ had previous emergency dialysis initiated through internal jugular vein cannulation and 78 patients $(12.3 \%)$ had pre-emptive AV fistula creation and did not need IJV cannulation. Few patients were on intermittent dialysis (25 patients), 395 patients (62.3\%) were receiving twice weekly and 136 patients (21.5\%) were receiving thrice weekly haemodialysis. 59 patients $(9.3 \%)$ were positive for either viral marker (34 were HBSAg positive, 19 were HCV positive and 6 were HIV positive), 2 patients were positive for two (HBSAg and anti-HCV) and none for all three. Due to acute left ventricular failure, 19 patients required assisted ventilation and diuretics and also underwent emergency HD.

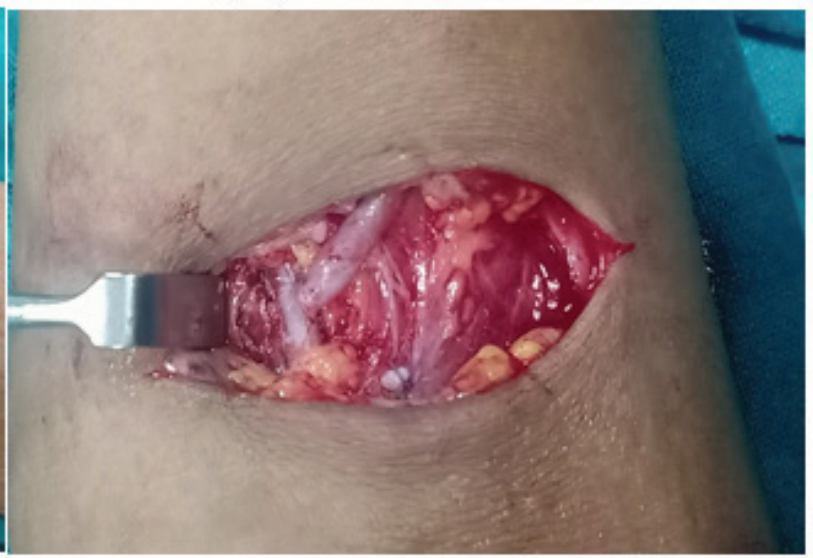

Clinical Photograph 4

\section{Results and Discussion}

Among the 634 patients, the most common anastomosis was Radio-cephalic (386 patients, 60.9\%) followed by Brachio-cephalic (186 patients, 29.3\%) and Transposed Basilic vein with Brachial artery (62 patients, 9.8\%). Among the Radio-cephalic group, the location was proximal forearm in $138(21.8 \%)$ patients and distal forearm in 248(39.1\%) patients.

Mean operating time was 10 minutes longer for the proximal forearm Radio-cephalic AVF group and 5 minutes longer for distal forearm Radio-cephalic AVF than the Brachio-cephalic and Brachio-basilic groups $(\mathrm{p}=0.018)$.

End-to-side anastomosis was the hands down choice over side-to-side anastomosis. Side to side anastomosis of Cephalic vein with Brachial artery was done in 5 patients and of Cephalic vein with Radial artery was performed in 2 cases but was abandoned because of the development of distal hyperaemia and oedema in all cases. On the contrary, distal oedema was seen in only $7 \%$ patients undergoing end-to-side anastomosis.

There was no difference in the AVF patency rate with respect to the suturing technique usedcontinuous versus interrupted, as long as the surgeon was experienced in micro-vascular techniques. 
Patency rate of AV Fistula at 1 week was 91.2\%, at 1 month was $84.6 \%$, at 6 months was $83.8 \%$ and at 1 year was $82.9 \%$, after creation. After 2 years $74.8 \%$ AVF's were still useful for dialysis. AVF failure on Day 1 was a rather disheartening occurrence and was seen in 15 patients and was unanimously related to small calibre veins (less than $1.5 \mathrm{~mm}$ ), not amenable to percutaneous intervention. In case of failure of radial AV fistula, brachial AVF is always feasible as a second native AVF hemodialysis access.
There was a meaningful co-relation between dialysis and arterio-venous patency $(\mathrm{p}=0.04)$, specifically the number of hemodialysis sessions per week. Dialysis could increase the fistula patency rate because of better homeostasis and also because of the use of the anticoagulant, Heparin, during dialysis. Patency rates were also higher in patients receiving anti-platelets due to cardiac morbidity or otherwise.

Table showing patency rates as in various types of AVF

\begin{tabular}{|c|c|c|c|c|c|c|}
\hline S.no. & Type of AV Fistula & $\begin{array}{c}\text { Patency at 1 } \\
\text { week (\%) }\end{array}$ & $\begin{array}{c}\text { Patency at 1 } \\
\text { month (\%) }\end{array}$ & $\begin{array}{c}\text { Patency at 6 } \\
\text { months (\%) }\end{array}$ & $\begin{array}{c}\text { Patency at } \\
\text { 1 year (\%) }\end{array}$ & $\begin{array}{c}\text { Patency at 2 } \\
\text { years (\%) }\end{array}$ \\
\hline 1 & Distal Radio-cephalic & 88.3 & 81.8 & 79.8 & 79.4 & 70.7 \\
\hline 2 & Proximal Radio-cephalic & 87.5 & 81.3 & 80.6 & 79.5 & 74.3 \\
\hline 3 & Brachio-cephalic & 95.6 & 88.9 & 88.6 & 86.4 & 78.5 \\
\hline 4 & Brachio-basilic & 93.2 & 86.4 & 86.4 & 85.6 & 75.9 \\
\hline
\end{tabular}

There was no short term difference in the outcomes of patients receiving anti-platelet medication and those who did not, Over the long term however, patency rates of patients receiving anti-platelets were better $(p<0.05)$. This was even more significant in patients with radial AV Fistulas.

\begin{tabular}{|c|c|c|c|c|c|}
\hline \multirow{2}{*}{ S.no. } & \multirow{2}{*}{ Type of AV Fistula } & \multicolumn{2}{|c|}{ Patency at 1 month } & \multicolumn{2}{c|}{ Patency at 1 year } \\
\cline { 3 - 6 } & With Aspirin & No Aspirin & With Aspirin & No Aspirin \\
\hline 1 & Distal Radio-cephalic & 83.3 & 80.7 & 82.5 & 76.1 \\
\hline 2 & Proximal Radio-cephalic & 82.1 & 80.9 & 81.9 & 77.1 \\
\hline 3 & Brachio-cephalic & 89.2 & 88.6 & 88.7 & 83.9 \\
\hline 4 & Brachio-basilic & 87.4 & 85.4 & 88.5 & 82.7 \\
\hline
\end{tabular}

Patients with feeble or absent thrill in AV fistula were taken up for percutaneous intervention by the cardiologist. Of the 116 such patients, 59 were amenable to balloon dilatation. The return of blood flow rate sufficient for dialysis was maintained in 42 patients after 1 month. 
Mean duration of hospital stay was 1.08 days which was not significantly different for brachial and radial groups

Minor bleeding episodes which did not require any intervention were seen in 55(8.6\%) patients. The incidence was not significantly more in either group. Major bleeding episodes were seen in 19(3\%) patients and required intervention. Surgical exploration was done in 10 patients out of which only one patient required repair of anastomotic rupture in a too tight anastomosis. The other 9 patients had diffuse bleeding in the surgical wound possibly due to altered coagulation profile and platelet dysfunction which are common in ESRD patients. All patients with major bleeding episodes needed blood-work for coagulation profile, FFP and platelet transfusions, Protamine, PRBC transfusion and haemodialysis. It is probably a better idea to manage all major bleeding using medical intervention rather than surgical, if there is no doubt about the anastomosis and surgical haemostasis. Patients with bleeding had longer hospital stays (2-3 days).

Prolonged serous discharge from the surgical site was observed in 27 patients, sometimes for up to 2 months after surgery. It is probably related to more accommodative extra-cellular tissue in ESRD patients. This might require more frequent dressing changes, and over extended periods of time.

Hyperemia and upper limb swelling were seen in $7 \%$ patients more than three-fourths of which belonged to the brachial group. The incidence of these complications was much higher in the brachial group than in the radial group. All patients $(n=7)$ with sideto-side anastomosis developed extensive upper limb swelling in distal aspect.

Aneurysm of anastomosis and recipient vein were seen in $19(3 \%)$ patients. This was a delayed complication seen after a mean of 215.7 days after the surgery. 2 patients had a delayed rupture of the aneurysmal vein with episodes of major bleeding requiring emergency surgery. Emergency ligation of the recipient vein just proximal to the anastomosis was done. Elective AV Fistula closure was also done in 6 patients who had excessively dilated veins with imminent rupture and 3 patients who had excessive upper limb swelling due to the aneurysmal veins. Despite the complication, however, most patients opt for continuing dialysis through the aneurysmal vein.

In the long run, blood-flow rates in a wellfunctioning AVF can exceed $1000 \mathrm{ml} / \mathrm{min}$ or more-a dramatic increase when compared with a blood flow rate of $20-30 \mathrm{ml} / \mathrm{min}$ in a normal peripheral artery and the immediate post-operative flow rate of $200-300 \mathrm{ml} /$ min after opening of the fistula. This is accompanied by a decrease in peripheral resistance. The adaptive venous response is clearly the consequence of increased flow which in turn depends on arterial dilatation which is the crucial and often limiting step $\left[^{2}\right]$

\section{Conclusion}

Even though 'End stage renal disease' is more common in females, most patients undergoing Renal Replacement Therapy are males. The average age of patients getting afflicted with renal dysfunction has shown a steady downward trend, over the years. Radio-cephalic site in distal forearm was the most common location for arterio-venous fistula creation. End-to-side anastomosis was the hands down choice over side-to-side anastomosis.

In seasoned hands, with good experience in micro-vascular surgery, patency rates of AV fistulas are high and provide adequate flow rates for years. The outcome can be improved further by using low dose Aspirin(75-150 mg OD). Complication rates are low and outrun the benefits by miles. Hyperaemia and upper limb swelling were much more common 
in the brachial group than in the radial group. Minor bleeding episodes not requiring intervention were 3 times more common than major bleeding episodes. Surgical exploration for bleeding is indicated only in rare patients who have very large volume of bleeding due to anastomotic rupture. Aneurysm of anastomosis and recipient vein were seen in 3\% patients. Only patients with impending rupture or associated upper limb swelling or intractable pain go in for AVF closure, after creation of a second AV fistula in the other upper limb. Overall complication rates are very low.

Ethical Clearence- Taken from Ethical Committee, SGRRIHMS, Dehradun

\section{Source of Funding- Self}

\section{Conflict of Interest- None}

\section{References}

1. Bharati J, Jha V. Global Dialysis Perspective: India. Kidney360 October 2020, 1 (10) 1143-1147.

2. Konner K. The anastomosis of the arteriovenous fistula-common errors and their avoidance. Nephrology Dialysis Transplantation, Volume 17, Issue 3, 1 March 2002, Pages 376-379.

3. Fokou M, Teyang A, Ashuntantang G, Kaze F, Eyengav VC, Mefire AC, Angwafo F. Complications of arteriovenous fistula for hemodialysis: an 8-year study. Ann Vasc Surg. 2012 Jul;26(5):680-4.

4. Smith GE, Gohil R, Chetter IC. Factors affecting the patency of arteriovenous fistulas for dialysis access. J Vasc Surg. 2012 Mar;55(3):849-55.

5. Wu CC, Jiang H, Cheng J, Zhao LF, Sheng KX, Chen JH. The outcome of the proximal radial artery arteriovenous fistula. J Vasc Surg. 2015 Mar;61(3):802-8.

6. Chang TI, Chen CH, Hsieh HL, Chen CY, Hsu SC, Cheng HS, Huang WC, Sue YM, Hsu YH, Lin FY, Shih CM, Lin SJ, Huang PH, Liu CT. Effects of cardiovascular medications on primary patency of hemodialysis arteriovenous fistula.
Sci Rep. 2020 Jul 22;10(1):12135.

7. Kucey AS, Joyce DP, O'Neill T, Fulton GJ, Plant WD, Manning BJ. Patients referred for arteriovenous fistula construction: a retrospective outcome analysis. Ir J Med Sci. 2020 May;189(2):685-691.

8. Quencer KB, Arici M. Arteriovenous Fistulas and Their Characteristic Sites of Stenosis. American Journal of Roentgenology. 2015;205: 726-734. 10.2214/AJR.15.14650

9. Yen CC, Tsai CF, Luo YY, Yang HY, Liu MY, Hung $\mathrm{PH}$, Hsu YH. Factors affecting fistula failure in patients on chronic hemodialysis: a population-based case-control study. BMC Nephrol. 2018 Aug 22;19(1):213.

10. Ayez N, Fioole B, Aarts RA, van den Dorpel MA, Akkersdijk GP, Dinkelman MK, de Smet AA. Secondary interventions in patients with autologous arteriovenous fistulas strongly improve patency rates. J Vasc Surg. 2011 Oct;54(4):1095-9.

11. Fitzgerald JT, Schanzer A, Chin AI, McVicar JP, Perez RV, Troppmann C. Outcomes of upper arm arteriovenous fistulas for maintenance hemodialysis access. Arch Surg. 2004 Feb;139(2):201-8.

12. Vo T, Tumbaga G, Aka P, Behseresht J, Hsu J, Tayarrah M. Staple aneurysmorrhaphy to salvage autogenous arteriovenous fistulas with aneurysm-related complications. J Vasc Surg. 2015 Feb;61(2):457-62.

13. $\mathrm{Yu} \mathrm{Q}, \mathrm{Yu} \mathrm{H}$, Chen $\mathrm{S}$, Wang L, Yuan W. Distribution and complications of native arteriovenous fistulas in maintenance hemodialysis patients: a single-center study. J Nephrol. 2011 Sep-Oct;24(5):597-603.

14. Glass C, Porter J, Singh M, Gillespie D, Young $\mathrm{K}$, Illig K. A large-scale study of the upper arm basilic transposition for hemodialysis. Ann Vasc Surg. 2010 Jan;24(1):85-91

15. Son HJ, Min SK, Min SI, Park YJ, Ha J, Kim SJ. Evaluation of the efficacy of the forearm basilic vein transposition arteriovenous fistula. J Vasc 
Surg. 2010 Mar;51(3):667-72.

16. Cheng CT, Chang YC, Tam KW, Yen YC, Ko YC. Comparison between transposed Brachiobasilic fistula and Arteriovenous Graft for upper limb arteriovenous access in patients on hemodialysis. Vasc Endovascular Surg. 2021 Feb;55(2):164-170.
17. Ashwal AJ, Padmakumar R, Abdul Razak UK, Naveen Chandra GS. Percutaneous intervention for restoration of patency of occluded lower limb arteriovenous dialysis access. Indian Heart $\mathrm{J}$. 2018 May-Jun;70(3):450-454. 